\title{
A Novel Treatment Strategy by Natural Products in NLRP3 Inflammasome-Mediated Neuroinflammation in Alzheimer's and Parkinson's Disease
}

\author{
Jun Ho Lee ${ }^{1}$, Hong Jun Kim ${ }^{1}$, Jong Uk Kim ${ }^{1}$, Tae Han Yook ${ }^{1}$, Kyeong Han Kim ${ }^{1}$, Joo Young Lee ${ }^{2}$ (D) \\ and Gabsik Yang ${ }^{1, *}$ \\ 1 College of Korea Medicine, Woosuk University, Jeonju-si, Jeollabuk-do 54986, Korea; \\ celtece@daum.net (J.H.L.); kimboncho@woosuk.ac.kr (H.J.K.); ju1110@hanmail.net (J.U.K.); \\ nasiss@naver.com (T.H.Y.); solip922@hanmail.net (K.H.K.) \\ 2 BK21plus Team, College of Pharmacy, The Catholic University of Korea, Bucheon 14662, Korea; \\ joolee@catholic.ac.kr \\ * Correspondence: yanggs@woosuk.ac.kr; Tel.: +82-63-290-9030
}

Citation: Lee, J.H.; Kim, H.J.; Kim, J.U.; Yook, T.H.; Kim, K.H.; Lee, J.Y.; Yang, G. A Novel Treatment Strategy by Natural Products in NLRP3 Inflammasome-Mediated Neuroinflammation in Alzheimer's and Parkinson's Disease. Int. J. Mol. Sci. 2021, 22, 1324. https://doi.org/ $10.3390 /$ ijms 22031324

Academic Editor: Dong-Sung Lee Received: 12 January 2021

Accepted: 23 January 2021

Published: 28 January 2021

Publisher's Note: MDPI stays neutral with regard to jurisdictional claims in published maps and institutional affiliations.

Copyright: (c) 2021 by the authors. Licensee MDPI, Basel, Switzerland. This article is an open access article distributed under the terms and conditions of the Creative Commons Attribution (CC BY) license (https:/ / creativecommons.org/licenses/by/ $4.0 /)$.

\begin{abstract}
Alzheimer's disease (AD) and Parkinson's disease (PD) are the most common neurodegenerative diseases. Many studies have demonstrated that the release of NLRP3 inflammasome-mediated proinflammatory cytokines by the excessive activation of microglia is associated with the pathogenesis of $\mathrm{AD}$ and PD and suggested that the NLRP3 inflammasome plays an important role in $\mathrm{AD}$ and PD development. In both diseases, various stimuli, such as $\mathrm{A} \beta$ and $\alpha$-synuclein, accelerate the formation of the NLRP3 inflammasome in microglia and induce pyroptosis through the expression of interleukin (IL)-1 $\beta$, caspase-1, etc., where neuroinflammation contributes to gradual progression and deterioration. However, despite intensive research, the exact function and regulation of the NLRP3 inflammasome has not yet been clearly identified. Moreover, there have not yet been any experiments of clinical use, although many studies have recently been conducted to improve treatment of inflammatory diseases using various inhibitors for NLRP3 inflammasome pathways. However, recent studies have reported that various natural products show improvement effects in the in vivo models of AD and PD through the regulation of NLRP3 inflammasome assembly. Therefore, the present review provides an overview of natural extraction studies aimed the prevention or treatment of NLRP3 inflammasome-mediated neurological disorders. It is suggested that the discovery and development of these various natural products could be a potential strategy for NLRP3 inflammasome-mediated AD and PD treatment.
\end{abstract}

Keywords: NLRP3 inflammasome; Alzheimer's disease; Parkinson's disease; natural products

\section{Introduction}

Neuroinflammation, an inflammatory response that occurs in the damaged central nervous system, is an important factor in neurodegeneration. Various types of cells, including microglia, astrocytes, and macrophages, are involved in this complicated inflammatory process. Microglia activated by pathological triggers migrate to the location of the injury and stimulate an innate immune response, including the production of pro-inflammatory cytokines, resulting in neuronal death [1,2]. In addition, inflammatory mediators produced by activated microglia promote astrogliosis, which in turn amplifies the inflammatory response by the additional secretion of inflammatory mediators. Persistent inflammation that occurs during the pathogenesis of various neurological disorders in the central nervous system is mediated by activation of the NLRP3 inflammasome, a key innate immune sensor that is associated with the progression of several neurological disorders, including Alzheimer's disease (AD), Parkinson's disease (PD), traumatic brain injury, stroke, depression, and multiple sclerosis [3,4]. 
Currently, there are only five types of medicine for AD treatment (four types of cholinesterase inhibitor and one type of N-methyl-D-aspartate receptor blocker), and 13 types of medicine for PD treatment (two types of dopaminergic receptor blocker, five types of dopamine agonist, three types of monoamine oxidase inhibitor, three types of catechol O-methyltransferase inhibitor) which are approved by the U.S. Food and Drug Administration. However, these treatments that have been developed so far only slightly delay the progression of the disease and have limited efficacy for early-stage patients, lack an efficient drug delivery system, and have various effects $[5,6]$.

In recent years, the drug mechanisms of the developed therapeutic agents have been expanding into inflammation, neuroprotection, vascular factors, metabolism, and neuroregeneration [7]. The development of a natural product-derived therapeutic agent can avoid the side effects of existing medicines and enable a wide range of drug mechanisms to be found. Therefore, in this review, we summarize the contribution of the mechanism of neuroinflammation contributes to $\mathrm{AD}$ and $\mathrm{PD}$ development, and then describe the activation of the NLRP3 inflammasome and its pathogenic role in AD and PD. In addition, we attempt to update the current knowledge of the neuroprotective effects of natural products on AD and PD treatment through regulation of the NLRP3 inflammasome. Therefore, this review attempts to provide a new perspective and potential strategy for reducing the development and progression of neurological disorders through the regulation of natural products in the NLRP3 inflammasome.

\section{Mechanism of NLRP3 Inflammasome Activation and Regulation}

The NLRP3 inflammasome is the most recently studied inflammatory control complex (Figure 1). It is activated by various stimuli and it contributes to the pathology of inflammatory diseases. Despite extensive investigation, the mechanism of NLRP3 inflammasome activation remains unclear. However, it is known that NLRP3 inflammasome activation requires two signaling steps provided by several exogenous and endogenous activators. The first signal (priming signal) is the nuclear factor kappa B (NF- $\mathrm{kB}$ )-dependent transcription of NLRP3 and pro-IL-1 $\beta$, which is triggered by the binding of the Toll-like receptor (TLR)4 ligand lipopolysaccharide (LPS) to the receptor [8-10]. The signal then promotes the expression of inflammasome components, including NLRP3, procaspase-1, and pro-IL$1 \beta$ (Figure 1). The first signal is initiated by TLR4 and then relayed by relevant adapter molecules, including myeloid differentiation factor 88 (MyD88), IRAK1, and IRAK4, without the requirement for new protein synthesis [11-13]. The second signal (activation signal), involved in the assembly and activation of the NLRP3 complex, is induced by extracellular ATP, certain bacterial toxins, and various types of crystalline and particulate matter. This signal subsequently produces active cleaved caspase- 1 . When microglia are activated by the NLRP3 activator as part of the second signal, NLRP3 undergoes oligomerization through homologous NACHT domain interactions, which recruits the PYD domain to interact with the PYD of ASC that triggers ASC fibrillar binding. Next, the ASC aggregate recruits CARD, which interacts with the CARD domain of procaspase- 1 to promote caspase- 1 activation and the subsequent polymerization of ASC fibrils into a large fibrous protein complex called ASC speck $[14,15]$. Clustered procaspase- 1 mediates self-cleavage and activation in the form of activated caspase- 1 , which cleaves the precursors of IL-1 $\beta$ and IL-18 to produce activated forms of IL-1 $\beta$ and IL-18 (Figure 1), triggering an inflammatory response and pyroptosis $[16,17]$. In this process, the post-translational modifications of NLRP3, such as deubiquitination and phosphorylation, are necessary for the assembly and activation of the NLRP3 inflammasome (second signal) $[12,18]$. However, the exact mechanism by which NLRP3 inflammasome assembly is promoted is still unknown. When the activation of NLRP3 is triggered, the formation of ASC speck is considered an upstream indication of NLRP3 activation [19]. 


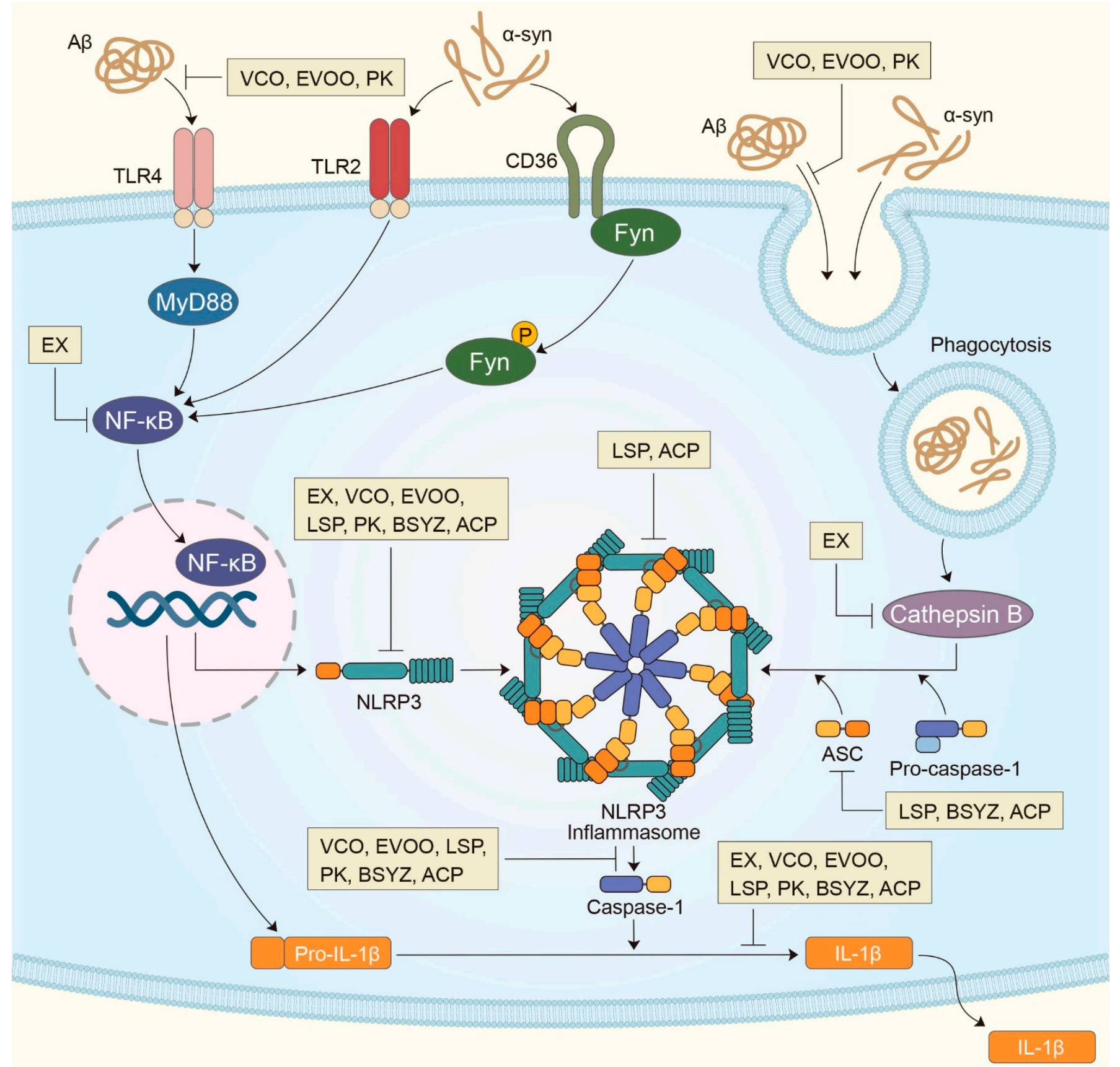

Figure 1. Mechanism of NLRP3 inflammasome activation in AD and PD with potential intervention of natural extractions. In the transcriptional NLRP3 inflammasome activation, A $\beta$ recognized by toll-like receptor 4 (TLR4) in microglia activates the myeloid differentiation primary response protein MyD88 (MYD88)-nuclear-factor- $\mathrm{B}$ (NF- $\mathrm{kB}$ ) pathway, and $\alpha$-synuclein recognized by toll-like receptor 2 (TLR4) and CD36 in microglia activates the Fyn kinase-mediated NF- $k B$ pathway to elevate IL-1 $\beta$ and the NLRP3 expression. Along with the expression of NLRP3 following NF- $\mathrm{B}$ translocation, lysosome leakage is induced by the phagocytosis of $A \beta$ and $\alpha$-syn resulted in the emission of cathepsin $B$, which in turn induces the NLRP3 inflammasome complex consisting of NLRP3, ASC and pro-casparase- 1 . The release of IL- $1 \beta$ promoted by active caspase- 1 can cause a continuous inflammatory response that contributes to neurodegeneration in AD and PD. Meanwhile, current studies have shown that various natural extractions are able to prevent or treat in AD and PD through regulation of the NLRP3 inflammasome/caspase $1 /$ IL-1 $\beta$ axis. These natural extractions can inhibit NLRP3 inflammasome activation in microglia, thereby the secretion of IL-1 $\beta$ was suppressed. ACP, Antrodia comphorata; BSYZ, Bushen-Yizhi formula; EVOO, Oleocanthal-rich extra-virgin olive oil; EX, Epimedii Folium and Curculiginis Rhizoma; LSP, Lychee seed polyphenol; PK, Picrorhiza kurroa; VCO, Virgin coconut oil. 
In addition, potassium efflux, mitochondrial dysfunction, reactive oxygen species (ROS), and lysosome destruction were also identified as higher signaling events for NLRP3 inflammasome activation. Most NLRP3 stimuli can cause the outflow of potassium from cells. Conversely, the inhibition of potassium efflux by high extracellular potassium concentration blocks most stimulus-induced NLRP3 inflammasome activation. Thus, potassium efflux is a major upstream signaling event for NLRP3 inflammasome activation (Figure 1). In contrast, the role of mitochondria and ROS in NLRP3 inflammasome activation remains controversial. Numerous studies support the role of mitochondrial dysfunction and associated ROS in NLRP3 inflammasome activation. However, other studies have shown that ROS is only required to prime the NLRP3 inflammasome and that mitochondrial dysfunction is indispensable for NLRP3 inflammasome activation. In addition, lysosome damage is only required for NLRP3 inflammasome activation by particulate matter. Several studies have suggested that members of the cathepsin family released from damaged lysosomes mediate NLRP3 inflammasome activation (Figure 1). In addition to these upstream events, many NLRP3 interacting partners and post-translational variants of NLRP3 regulate NLRP3 inflammasome activation [20].

\section{Role of NLRP3 Inflammasome in AD}

NLRP3 inflammasome activation has been implicated in the pathogenesis of neurodegenerative diseases, especially AD and PD. The deposition of misfolded amyloid- $\beta$ (A $\beta$ ) in the brain is a major pathological event in AD. Recent studies have shown that NLRP3 inflammasome-mediated neuroinflammation plays an important role in the pathogenesis of AD [21]. The secretion of IL- $1 \beta$ by A $\beta$ was dependent on NLRP3, ASC, and caspase- 1 activities and required cathepsin $B$ released from damaged lysosomes. Furthermore, fewer microglia and mononuclear phagocytes were recruited to $A \beta$ in the brains of ASC and caspase-1 knockout mice (IL-1 receptor- or MyD88-deficient mice) after the injection of A $\beta$ into the striatum compared with wild-type controls. The intra-hippocampal injection of ASC speck into APP/PS1 mice promoted A $\beta$ plaque formation and accumulation. However, it was not possible to induce the spread of A $\beta$ pathology in ASC-deficient APP/PS1 mice [22]. Furthermore, abnormal microglia-specific NLRP3 activation induced chronic neuro-inflammation in the pathological process of $\mathrm{AD}$, resulting in microglia $\mathrm{A} \beta$ phagocytic dysfunction, peripheral nerve cell damage, and severe pathological damage [23]. However, this process can be altered by the microglia-specific destruction of the NLRP3 inflammasome. In addition, excessive NLRP3 activation and elevated IL-1 $\beta$ levels in microglia can exacerbate neural tau hyper-phosphorylation, neurofibrillary tangles, and synaptic dysfunction in AD induced by a detrimental chronic inflammatory response [24-27]. ASC or NLRP3 deficiency was reported to reduce tau pathology and protect against cognitive impairment in tau transgenic mice $[28,29]$. In addition, IL- $1 \beta$ inhibition in a triple transgenic ( $3 \times \mathrm{Tg}$ ) mouse model of AD induced cognition recovery, tau pathology attenuation, and neuronal beta-catenin pathway function recovery [30,31]. Furthermore, regulation of the NLRP3 inflammasome reduced the inflammatory response, which led to an alleviation of the pathological process of AD. Another study showed that inhibition of the NLRP3 inflammasome reduced $\mathrm{A} \beta$ deposition, neuro-inflammation, and cognitive impairment in an AD mouse model [32-35]. Thus, these findings suggest that A $\beta$-induced NLRP3 inflammasome activation promotes the pathogenesis of $\mathrm{AD}$ by triggering the release of pro-inflammatory cytokines and nerve inflammation. Consistently, brain samples from human AD patients have shown increased caspase- 1 activity compared with age-matched control patients without dementia [2].

\section{Natural Extraction Treatment Studies for NLRP3 Inflammasome Regulation in AD}

As mentioned above, microglial NLRP3 inflammasome activation is an important characteristic of AD pathogenesis. Several mouse model studies have shown that NLRP3 or caspase- 1 deficiency markedly reduced the $A \beta$ burden and reduced cognitive impairment $[36,37]$. In addition, IL-1 $\beta$ inhibition significantly reduced brain nerve inflamma- 
tion, alleviated cognitive impairment, and partially reduced $\mathrm{A} \beta$ deposition in 3xTg-AD mice $[30,31,38]$. These findings suggest that novel therapeutic interventions at the molecular level might modulate AD pathology through the inhibition of NLRP3 inflammasome activation. Therefore, therapeutic strategies of inflammasome constructs or downstream signal regulation might reduce neurological inflammation and slow the progression of AD. Here, we present recent studies of natural extraction-mediated NLRP3 inflammasome regulation (Table 1).

Epimedii Folium is the dried leaf of Epimedium brevicornu Maxim, and Curculiginis Rhizoma is the dried rhizoma of Curculigo orchioides Gaertn. In previous studies, Epimedii Folium and Curculiginis Rhizoma (EX) were shown to possess a wide range of neuro-protective, antiinflammatory, and antioxidant effects. Epimedii Folium and Curculiginis Rhizoma reduced the content of pro-inflammatory cytokines including tumor necrosis factor-alpha (TNF- $\alpha$ ), IL-1 $\beta$, and IL- 6 in the hippocampus and cortex of A $\beta$-induced rats. EX also reduced the levels of malondialdehyde and increased superoxide dismutase, catalase, glutathione, and glutathione peroxidase in serum. Furthermore, immunohistochemical analysis showed that EX inhibited the expression of NLRP3 and the phosphorylation of MAPKs, NF- $\mathrm{kB}$, MyD88, and cathepsin B [38].

Virgin coconut oil (VCO) is rich in natural antioxidants that regulate lipid profiles and has anti-inflammatory and antioxidant activity, which might be effective for the treatment and prevention of AD. VCO normalized NLRP3 inflammasome gene expression and oxidative stress compared with an A $\beta$ - and high fat diet HFD-induced AD rat model. Congo Red and Cresyl Violet staining, as well as immunohistochemistry showed that VCO improved hippocampus histological changes by reducing $A \beta$ plaques and phosphorylated tau $[34,39]$.

Extra-virgin olive oil (EVOO) protected mice from $A \beta$-related pathology by restoring the blood-brain barrier (BBB) function at an early age before pathology onset. Oleocanthal $(\mathrm{OC})$, a phenolic compound abundant in EVOO, restored BBB function, reduced $A \beta$ brain levels, and reduced inflammation, as evidenced by reduced astrogliosis and brain levels of cytokines. OC-rich EVOO restored BBB function and reduced AD-associated pathology by reducing neuroinflammation through the inhibition of NACHT, leucine-rich repeats (LRR), and the NLRP3 inflammasome and inducing autophagy [35].

Lychee seeds contain many polyphenols, including rutin, quercetin, catechin, and proanthocyanidins, which inhibited $A \beta$-induced apoptosis in neuronal cells and the neuroinflammatory response in microglia via the NF- $\mathrm{KB}$ pathway. Lychee seed polyphenols (LSPs) significantly decreased monolayer permeability, inhibited NLRP3 inflammasome activation in $\mathrm{A} \beta_{25-35}$-induced bEnd. 3 cells, and induced autophagy via the AMPK/mTOR/ULK1 pathway. In addition, LSPs improved spatial learning and memory function, increased expression of tight junction proteins (TJ), and inhibited the expression of NLRP3, caspase-1, IL-1 $\beta$, and p62 in APP/PS1 mice. In another study, LSPs also suppressed activation of the NLRP3 inflammasome by inhibiting the expression of NLRP3 and ASC, the cleavage of caspase- 1 , and the release of IL- $1 \beta$ in A $\beta_{1-42}$-induced BV- 2 cells and recovered PC-12 cells that were induced by incubated medium from $A \beta_{1-42}$-treated BV-2 cells. Moreover, LSPs improved cognitive function and inhibited NLRP3 inflammasome activation in APP/PS1 mice [40,41].

Picrorhiza kurroa Bentham, Scrophulariae (PK) is a commonly used herb in Ayurvedic medicine, and several studies reported its therapeutic effects for various diseases related to its antioxidant, anti-inflammatory, anti-allergic, and anti-asthma effects. In a mouse model of AD, PK altered disease-related microglial neuro-inflammation, as evidenced by shifting microglia phenotypes from the inflammatory form to the anti-inflammatory form and inhibiting the nucleotide-binding oligomerization domain, leucine-rich repeat, and NLRP3 inflammasome activity. Moreover, PK induced silent information regulator type $1 /$ peroxisome proliferator-activated receptor- $\gamma$ signaling, decreasing the expression of $\beta$-secretase 1, which is involved in $A \beta$ production [39]. 


\section{Role of NLRP3 Inflammasome in PD}

Recent studies have also reported a role for the NLRP3 inflammasome in PD. The three features of PD are neuro-inflammation, the gradual loss of dopaminergic neurons in the SNPc and striatum, and the associated accumulation of protein aggregates rich in $\alpha$-synuclein in the Lewy form of disease [42]. $\alpha$-synuclein acts as a damage-associated molecular pattern and alters microglial TLR expression. An increase in the number of classically activated microglia prior to neuronal loss was reported in a mouse model of $\alpha$-synuclein overexpression [43]. In 2018, Gordon et al. observed that the activation of microglial NLRP3 inflammasomes could be triggered by the presence of fibrillar $\alpha$-synuclein and the absence of $\alpha$-synuclein-mediated dopaminergic neurons. Tissue samples collected from the substantia nigra of brains from patients with PD showed elevated levels of cleaved caspase- 1 and ASC. The expression of NLRP3 in activated microglia was also observed in post-mortem tissue lysates. In addition, in a mouse model of PD, microglial NLRP3 activation by $\alpha$-synuclein was delayed without mediating pyroptosis but caused potent IL-1 $\beta$ and ASC release [44]. Very recently, Panicker et al. revealed an association between misfolded $\alpha$-synuclein and the activation of microglial inflammation in PD. Previously, they reported that microglia Fyn kinase was rapidly activated by LPS and TNF- $\alpha$. However, in vitro and in vivo studies showed that the non-receptor tyrosine kinase, Fyn kinase, regulated the microglial uptake of misfolded $\alpha$-synuclein and subsequent activation of the NLRP3 inflammasome. Fyn kinase regulates NLRP3 inflammasome activation by regulating the protein kinase $C(\mathrm{PKC}) \delta$-dependent nuclear translocation of NF- $\mathrm{kB}$ p65, promoting ROS generation, and the introduction of $\alpha$-synuclein, which promotes subsequent NLRP3 inflammasome activation, thereby regulating the LPS-dependent priming of the microglial NLRP3 inflammasome $[45,46]$. Overall, $\alpha$-synuclein aggregation and the microglial NLRP3 inflammasome appear to be involved in a vicious cycle of neuronal cell death mediated via high levels of cytokine production during the onset of PD. This demonstrates that the link between $\alpha$-synuclein and microglial NLRP3 activation is a source of persistent neuroinflammation that can lead to gradual neuronal loss in PD.

\section{Natural Extraction Treatment Studies for NLRP3 Inflammasome Regulation in PD}

Due to the lack of information on how the NLRP3 structure and its activation directly modulate dopaminergic neuronal loss, therapeutic strategies involving selective microglial NLRP3 modulators that could be used as novel therapeutics for PD treatment are studied less than those for AD. Here, we present the results of recent studies of NLRP3 inflammasome-based therapeutic development of natural extractions for AD (Table 1).

Table 1. NLRP3 inflammasome targeting study of natural extraction in Alzheimer's disease (AD) and Parkinson's disease (PD).

\begin{tabular}{|c|c|c|c|c|c|}
\hline Origin of Extraction & Target Indication & $\begin{array}{c}\text { Cell or Animal } \\
\text { Model }\end{array}$ & Inducer & Mode of Action and Target Signal & Ref. \\
\hline $\begin{array}{l}\text { Epimedii Folium and } \\
\text { Curculiginis } \\
\text { Rhizoma }\end{array}$ & $\mathrm{AD}$ & SD rats & $\mathrm{A} \beta_{1-42}$ & $\begin{array}{c}\downarrow \quad \text { TNF- } \alpha, \text { IL-1 } \beta, \text { IL-6, MDA, SOD, } \\
\text { CAT, GSH, GSH-Px, NLRP-3, ASC, } \\
\text { caspase-1, MAPKs, NF-kB, MyD88, } \\
\text { cathepsin B } \\
\uparrow \quad \text { Spatial learning and memory } \\
\text { ability }\end{array}$ & [38] \\
\hline Virgin coconut oil & $\mathrm{AD}$ & Wistar rats & $\mathrm{A} \beta_{1-40}, \mathrm{HFD}$ & $\begin{array}{c}\downarrow \text { TAC, TOS, glutathione, IL-1 } \beta, \\
\text { caspase-1, NLRP3, A } \beta \text { plaque } \\
\text { formation, phosphorylated tau } \downarrow \\
\text { Spatial memory and learning ability, } \\
\text { cell density in CA1 region }\end{array}$ & [34] \\
\hline $\begin{array}{l}\text { Oleocanthal-rich } \\
\text { extra-virgin olive oil }\end{array}$ & $\mathrm{AD}$ & TgSwDI mice & Transgenes & 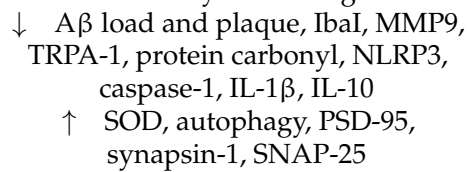 & [35] \\
\hline
\end{tabular}


Table 1. Cont.

\begin{tabular}{|c|c|c|c|c|c|}
\hline Origin of Extraction & Target Indication & $\begin{array}{l}\text { Cell or Animal } \\
\text { Model }\end{array}$ & Inducer & Mode of Action and Target Signal & Ref. \\
\hline \multirow[t]{2}{*}{$\begin{array}{l}\text { Lychee seed } \\
\text { polyphenol }\end{array}$} & \multirow[t]{2}{*}{$\mathrm{AD}$} & $\begin{array}{l}\text { BV-2, PC-12 cells } \\
\text { APP/PS1 mice }\end{array}$ & $\begin{array}{c}\mathrm{A} \beta_{1-42} \\
\text { Transgenes }\end{array}$ & $\begin{array}{cc}\downarrow & \text { NLRP3, ASC, caspase-1, IL-1 } \beta \text {, cell } \\
\text { death, apoptosis, NLRP3 } \\
\text { inflammasome } \\
\uparrow \quad \text { Autophagy, cognitive function } \\
\downarrow \quad \text { NLRP3, caspase-1, ASC, IL-1 } \beta,\end{array}$ & \multirow[t]{2}{*}[40,41]{} \\
\hline & & $\begin{array}{l}\text { bEnd.3 cells } \\
\text { APP/PS1 mice }\end{array}$ & $\begin{array}{c}\mathrm{A} \beta_{25-35} \\
\text { Transgenes }\end{array}$ & $\begin{array}{c}\text { NLRP3 inflammasome } \\
\uparrow \quad \text { Cell viability and permeability, TJs, } \\
\text { autophagy, spatial learning and } \\
\text { memory function }\end{array}$ & \\
\hline Picrorhiza kurroa & $\mathrm{AD}$ & 5xFAD mice & Transgenes & $\begin{aligned} \downarrow \quad \mathrm{A} \beta & \beta \text { burden, IbaI, IL-1 } \beta, \text { NLRP3, } \\
& \text { caspase-1, C99, BACE1 } \\
& \text { Cognitive function, FIZZ1 }\end{aligned}$ & [39] \\
\hline $\begin{array}{l}\text { Bushen-Yizhi } \\
\text { formula }\end{array}$ & PD & $\begin{array}{l}\text { BV-2 cells } \\
\text { C57BL } / 6 \text { mice }\end{array}$ & $\begin{array}{l}\mathrm{MPP}^{+} \\
\text {MPTP }\end{array}$ & $\begin{array}{c}\downarrow \text { Motor impairment, dopaminergic } \\
\text { neurons neurodegeneration, NLRP3, } \\
\text { IL- } 1 \beta \text { caspase- } 1 \text {, ASC }\end{array}$ & [47] \\
\hline \multirow[t]{2}{*}{ Antrodia comphorata } & PD & C57BL/ 6 mice & 6-OHDA & $\begin{array}{c}\downarrow \quad \text { NLRP3 inflammasome } \\
\uparrow \quad \text { Neurobehavior, dopamine }\end{array}$ & \multirow[t]{2}{*}[48,49]{} \\
\hline & PD & $\begin{array}{l}\text { MES23.5 cells } \\
\text { C57BL/ } 6 \text { mice }\end{array}$ & 6-OHDA & $\begin{array}{cc}\downarrow & \text { NLRP3, ASC, IL-1 } \beta, \text { caspase-1, } \\
\text { ROS } \\
\uparrow \quad \text { Dopaminergic neuron protection }\end{array}$ & \\
\hline
\end{tabular}

Bushen-Yizhi formula (BSYZ) is a traditional Chinese medicine composed of common Cnidium fruit, tree peony bark, ginseng root, Radix Polygoni Multiflori Preparata, Barbary wolfberry fruit, and Fructus Ligustri Lucidi. BSYZ has extensive neuroprotective properties, including anti-senescence and anti-apoptosis effects, as well as affects alleviating oxidative stress in various AD animal models. A recent study confirmed that BSYZ alleviated motor impairment and dopaminergic neuron degeneration, attenuated microglia activation, inhibited NLPR3 activation, and decreased the levels of inflammatory cytokines in the 1-methyl-4-phenyl-1,2,3,6-tetrahydropyridine (MPTP)-induced mouse brain. Furthermore, BSYZ inhibited NLRP3 activation and IL-1 $\beta$ production of 1-methyl-4-phenyl-pyridinium (MPP+)-stimulated BV-2 microglia cells [47].

Antrodia camphorata polysaccharide (ACP) is the main component of the natural polyporaceae Aphididae. ACP, a unique polyporaceae fungus in Taiwan, is composed of a variety of monosaccharides. It is well known that various multiple polysaccharides have anti-inflammatory effects. A recent study showed that ACP inhibited the expression of ROS-NLRP3 induced by 6-OHDA, exerted a protective role in MES23.5 cells, reduced the activation level of ROS-NLRP3 in the substantia nigra-striatum, and improved the exercise capacity of PD mice. Another study confirmed that ACP elevated levels of dopamine in the striatum and decreased the expression of the NLRP3 inflammasome signal in the striatum of 6-OHDA-induced PD mice $[48,49]$.

\section{Discussion}

Neurodegenerative diseases, especially $\mathrm{AD}$ and $\mathrm{PD}$, are major medical problems and social issues worldwide. AD and PD account for the largest proportion of neurodegenerative diseases worldwide, and they were the neurological diseases that increased the most between 1990 and 2016 [50]. However, effective treatments that improve the clinical outcomes of patients with AD and PD have not been developed yet.

In recent years, many studies have shown the disease-improving effects of single compounds derived from natural extractions, such as fruits, vegetables, and Chinese herbal medicines, for the treatment of AD and PD, by regulating NLRP3 inflammasome assembly.

Baicalin, a flavonoid compound isolated from the root of Scutellaria baicalensis Georgi, has potent anti-inflammatory effects. In APP/PS1 transgenic mice, the oral administration of baicalin for 33 days significantly attenuated spatial memory dysfunction in the passive avoidance test and Morris water maze test. Although the reduction in $A \beta$ deposition was not significant, baicalin reduced microglia activation, IL-18, IL-1 $\beta$, and iNOS expres- 
sion in APP/PS1 mice and LPS/A $\beta$-stimulated BV2 cells. These results indicated the protective effects of baicalin in $\mathrm{AD}$ progression were related to the inhibition of microgliainduced neuroinflammation by suppressing the activation of NLRP3 inflammasomes and the TLR4/NF- $\mathrm{KB}$ signaling pathway [51].

Flavocoxid, a mixture of purified baicalin and catechin, has anti-inflammatory properties including inhibition of the peroxidase activity of COX enzymes, a common target of non-steroidal anti-inflammatory drugs. After the administration of flavocoxid to 3xTg-AD mice for 3 months, spatial learning and memory were significantly improved in the Morris water maze test, whereas amyloid deposit, eicosanoid production, neuron apoptosis, $\mathrm{p}$ $\mathrm{APP}$, and $\mathrm{p}$-tau were significantly reduced. In addition, flavocoxid reduced A $\beta$ plaques and neuronal loss in the hippocampus and isocortex of 3xTg-AD mice. In that study, the neuroprotective effects of flavocoxid were associated with the regulation of NLRP3 inflammasome-mediated IL-1 $\beta$ production [33].

Dihydromyricetin is an abundant flavanonol in Ampelopsis. A previous study reported that supplementation of dihydromyricetin for 4 weeks reduced microglia activation and expression of the NLRP3 inflammasome components, including NLRP3 and caspase-1, and A $\beta$ levels in APP/PS1 mice. Notably, dihydromyricetin significantly enhanced the conversion of BV-2 microglia from the M1 phenotype to the M2 phenotype, which may promote the clearance of $A \beta$ [52].

Resveratrol is a natural plant polyphenol compound abundant in red grape skin and wine. Resveratrol alleviated learning and memory impairments, and increased the expression of Sirt1, AMPK, and PKC- $1 \alpha$ in the hippocampus and prefrontal cortex of $A \beta 1-42$-induced $A D$ mice. In this $A D$ animal model, resveratrol significantly reduced $A \beta-$ mediated inflammatory responses by inhibiting NF- $\kappa$ B, IL-1 $\beta$, and NLRP3 expression [53].

Astaxanthin is a natural carotenoid abundant in marine organisms such as shrimp, crab, krill, salmon, and microalgae. In a previous study, supplementation of $0.2 \%$ astaxanthin in the diet of APP/PS1 mice for 60 days enhanced learning and memory in the radial eight-arm maze and Morris water maze tests, and reduced amyloid plaques, hyperphosphorylation of tau, microglia activation, and NLRP3 inflammasome assembly [54].

Tenuigenin (senegenin), a saponin isolated from Polygala tenuifolia root, is commonly used in Chinese medicine. Pre-treatment of MPTP-induced PD mice with tenuigenin for 10 days alleviated motor impairments, increased levels of dopamine and its metabolites in the striatum, reduced the loss of tyrosine hydrolase-positive neuronal cells, and attenuated the expressions of IL-1 $\beta$, caspase-1, and NLRP3 in the substantia nigra. In addition, treatment with tenuigenin reduced intracellular ROS and NLRP3-mediated inflammatory responses in microglia BV-2 cells [55].

Table 2 shows that these single compounds modulate the intracellular signaling cascade of the NLRP3 inflammasome/caspase-1/IL-1 $\beta$ axis in vivo to regulate inflammatory responses, alleviate oxidative stress, and improve symptoms of neurological disorders. However, there have been no clinical studies applying these experimental data. Although inflammasome downstream effectors including anakinra (IL-1 receptor antagonist), canakinumab (IL-1 $\beta$ neutralizing antibody), and rilonacept (soluble decoy receptor for IL-1 $\beta$ and IL-1 $\alpha$ ) have been approved as therapeutic agents [56], there have been no reports of their use in clinical trials of neurodegenerative diseases including AD and PD.

Table 2. NLRP3 inflammasome targeting study of single compound in AD and PD.

\begin{tabular}{|c|c|c|c|c|c|}
\hline Origin of Extraction & Target Indication & $\begin{array}{c}\text { Cell or Animal } \\
\text { Model }\end{array}$ & Inducer & Mode of Action and Target Signal & Ref. \\
\hline Flavocoxid & $\mathrm{AD}$ & $\begin{array}{l}\text { SH-SY5Y cells } \\
\text { APP/PS1 mice }\end{array}$ & $\begin{array}{c}\mathrm{A} \beta_{1-42} \\
\text { Transgenes }\end{array}$ & $\begin{array}{c}\downarrow \quad \text { Memory impairment, microglial } \\
\text { activation, IL-18, iNOS, IL-1 } \beta, \\
\text { caspase-3, apoptosis, NLRP3, } \\
\text { caspase-1, p-p65, p-IKB, TLR4 } \\
\downarrow \quad \mathrm{A} \beta \text { deposition, PGE-2, LTB-4, } \\
\text { NLRP3, IL-1 } \beta \text {, p-APP, presenilin-1, } \\
\quad \text { p-Tau, p-ERK 1/2, Bax } \\
\uparrow \quad \text { Cognitive functions, Bcl-2,Bcl-xL }\end{array}$ & [33] \\
\hline
\end{tabular}


Table 2. Cont.

\begin{tabular}{|c|c|c|c|c|c|}
\hline Origin of Extraction & Target Indication & $\begin{array}{c}\text { Cell or Animal } \\
\text { Model }\end{array}$ & Inducer & Mode of Action and Target Signal & Ref. \\
\hline Dihydromyricetin & $\mathrm{AD}$ & $\begin{array}{c}\text { BV-2 cells } \\
\text { APP/PS1 mice }\end{array}$ & $\begin{array}{c}\mathrm{A} \beta_{1-42} \\
\text { Transgenes }\end{array}$ & $\begin{array}{c}\downarrow \quad \mathrm{A} \beta \text { deposition, microglia } \\
\text { activation, NLRP3, caspase-1, IL-1 } \beta \text {, } \\
\text { TNF- } \alpha \text {, cognitive deficits } \\
\uparrow \quad \mathrm{A} \beta \text { clearance }\end{array}$ & [52] \\
\hline Resveratrol & $\mathrm{AD}$ & Kunming mice & $\mathrm{A} \beta_{1-42}$ & $\begin{array}{c}\downarrow \quad \text { Learning and memory } \\
\text { impairments, NF- } \mathrm{B} \text {, IL-1 } \beta \text {, NLRP3 } \\
\uparrow \quad \text { SIRT1, AMPK, PGC-1 } \alpha \text {, neuronal } \\
\text { integrity }\end{array}$ & [53] \\
\hline Astaxanthin & $\mathrm{AD}$ & $\mathrm{APP} / \mathrm{PS} 1$ mice & Transgene & $\begin{array}{c}\downarrow \quad \beta \text { deposition, nicastrin, NO, p-tau, } \\
\text { microglial activation, IL-1 } \beta \text {, TNF- } \alpha, \\
\text { Bcl-2, caspase-9, caspase-3, NLRP3, } \\
\text { ASC, caspase-1, IL-1 } \beta \\
\uparrow \text { BACE, SOD, p-GSK3 } \beta,\end{array}$ & {$[54,55]$} \\
\hline Tenuigenin & PD & $\begin{array}{l}\text { BV-2 cells } \\
\text { B6 mice }\end{array}$ & $\begin{array}{c}\text { LPS } \\
\text { 1-methyl-4-henyl- } \\
\text { 1,2,3,6- } \\
\text { Tetrahydropyridine }\end{array}$ & $\begin{array}{cc}\downarrow & \text { NLRP3, IL-1 } \beta, \text { caspase-1, ROS } \\
\uparrow & \text { Locomotor activity, dopamine, } \\
\text { tyrosine hydroxylase }\end{array}$ & [55] \\
\hline
\end{tabular}

Therefore, the discovery of inhibitors of NLRP3 inflammasome activation from bioactive compounds derived from natural substances might be a promising future strategy for the prevention and treatment of neurodegenerative diseases. Our study showed that $\beta$-carotene, sweroside, and sulforaphane [57-59], acting as NLRP3 inhibitors, might be applicable to the prevention of AD and PD.

In addition, knowledge regarding the neuroprotective properties of these bioactive compounds mediated through the regulation of the NLRP3 inflammasome is still lacking. Many candidate substances, such as the single compounds presented in Table 2, have been reported to have therapeutic effects. To better understand the role of the NLRP3 inflammasome in AD and PD, further studies on the activation mechanism of the NLRP3 inflammasome using more sophisticated animal model experiments and molecular-targeted NLRP3 treatments need to be performed.

In conclusion, there is extensive experimental evidence that NLRP3 inhibitors can modulate NLRP3 inflammasome activation to prevent or treat AD and PD; therefore, it would be of great value to discover various NLRP3 modulators and use them to develop new therapeutic strategies for treating neurodegenerative diseases. In addition, clinical studies of potential therapeutic agents are needed to study the detailed mechanisms that ultimately target the NLRP3 inflammasome in AD and PD.

\section{Conclusions}

In conclusion, this review summarizes the current knowledge of NLRP3 inflammasome activation in AD and PD. The NLRP3 inflammasome serves as an intracellular sensor for host-derived risk signals associated with neurological disorders. Especially, recent studies have shown much evidence that $\mathrm{AD}$ and $\mathrm{PD}$ are induced through the intracellular signaling cascade of the NLRP3 inflammasome/caspase- $1 /$ IL- $1 \beta$ axis. This evidence has shown that NLRP3 inflammation plays a crucial role in the development of $\mathrm{AD}$ and $\mathrm{PD}$.

In addition, this review presents evidence that several recent preclinical studies that numerous plant-derived chemicals can exert beneficial effects on AD and PD through various inhibitory functions on NLRP3 inflammasome assembly and activation. For decades, natural products have been regarded as potential substances for the prevention or treatment of cancer, metabolic and neurodegenerative diseases due to their antioxidant and anti-inflammatory activity. Based on the evidence summarized in this review, the use of natural products that act in various stages of NLRP3 signaling could be a pharmacological approach suitable for the management of $\mathrm{AD}$ and $\mathrm{PD}$ as well as chronic inflammatory conditions. 
However, since the etiological cause and mechanism of pathogenesis in $\mathrm{AD}$ and $\mathrm{PD}$ are not clearly identified, related research is still ongoing. In addition, in vivo models for $\mathrm{AD}$ and $\mathrm{PD}$ also have not been clearly established yet due to difficulties with proceeding to clinical trials. In particularly, there are currently no clinical trials showing the efficacy of natural products targeting NLRP3 in humans. Moreover, it is also necessary to further understand whether it is possible to inhibit the activation of the NLRP3 inflammasome at the transcriptional or post-transcriptional level or directly modify conformation. Nevertheless, the accumulated evidence has shown that the NLRP3 inflammasome plays an important role in the pathogenesis of various neuroinflammation and neurological disorders [60] and that natural products have an effect in the prevention and treatment of various inflammation prevention and inflammation-derived diseases. Therefore, natural product research is still needed through the regulation of NLRP3 inflammasome assembly in a wide range of neuropathies to identify various pharmacological mechanisms and develop therapeutic agents for such neuropathies.

Funding: This study was supported by a grant of the Korea Health Technology R\&D Project through the Korea Health Industry Development Institute (KHIDI), funded by the Ministry of Health \& Welfare, Republic of Korea (HF20C0145) and by grants from the National Research Foundation of Korea (NRF-2016R1C1B2016274, 2019R1C1007573 and NRF-2020R1I1A3072176) funded by the Korean government (Ministry of Science, ICT and Future Planning).

Conflicts of Interest: The authors declare no conflict of interest.

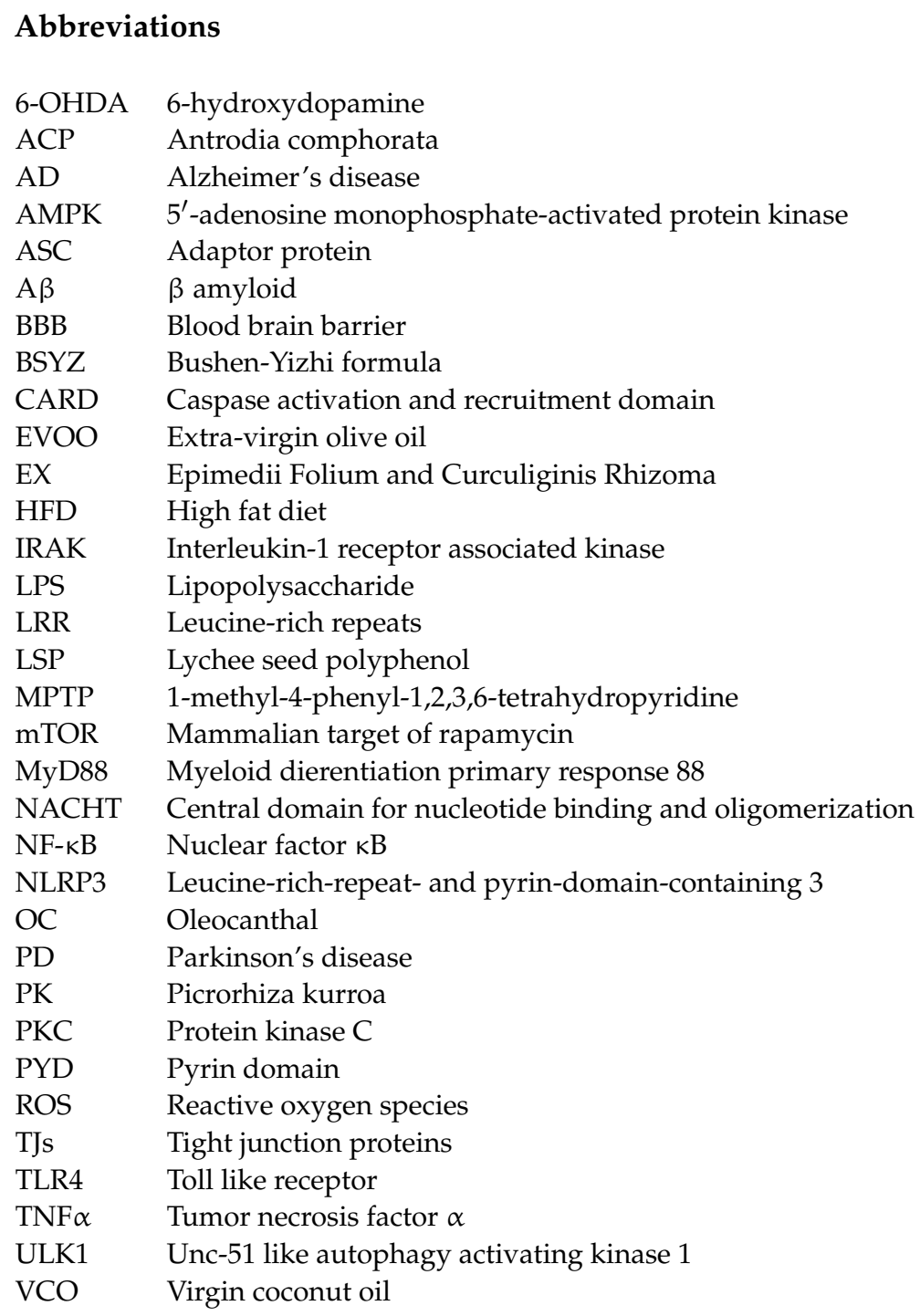




\section{References}

1. Mietto, B.S.; Mostacada, K.; Martinez, A.M. Neurotrauma and Inflammation: CNS and PNS Responses. Mediat. Inflamm. 2015, 2015, 1-14. [CrossRef]

2. Heneka, M.T.; McManus, R.M.; Latz, E. Inflammasome signalling in brain function and neurodegenerative disease. Nat. Rev. Neurosci. 2018, 19, 610-621.

3. Tong, Y.; Ding, Z.-H.; Zhan, F.-X.; Cai, L.; Yin, X.; Ling, J.-L.; Ye, J.-J.; Hou, S.-Y.; Lu, Z.; Wang, Z.-H.; et al. The NLRP3 inflammasome and stroke. Int. J. Clin. Exp. Med. 2015, 8, 4787-4794.

4. Kaufmann, F.N.; Costa, A.P.; Ghisleni, G.; Diaz, A.P.; Rodrigues, A.L.S.; Peluffo, H.; Kaster, M.P. NLRP3 inflammasome-driven pathways in depression: Clinical and preclinical findings. Brain Behav. Immun. 2017, 64, 367-383. [CrossRef]

5. Cummings, J.; Lee, G.; Ritter, A.; Sabbagh, M.; Zhong, K. Alzheimer's disease drug development pipeline: 2020. Transl. Res. Clin. Interv. 2020, 6, e12050. [CrossRef]

6. Rascol, O.; Payoux, P.; Ory, F.; Ferreira, J.J.; Brefelcourbon, C.; Montastruc, J.-L. Limitations of current Parkinson's disease therapy. Ann. Neurol. 2003, 53 (Suppl. 3), S3-S15. [CrossRef]

7. Tonda-Turo, C.; Origlia, N.; Mattu, C.; Accorroni, A.; Chiono, V. Current Limitations in the Treatment of Parkinson's and Alzheimer's Diseases: State-of-the-Art and Future Perspective of Polymeric Carriers. Curr. Med. Chem. 2018, 25, 5755-5771. [CrossRef]

8. Lamkanfi, M.; Dixit, V.M. Mechanisms and Functions of Inflammasomes. Cell 2014, 157, 1013-1022. [CrossRef]

9. Muñoz-Planillo, R.; Kuffa, P.; Martínez-Colón, G.; Smith, B.L.; Rajendiran, T.M.; Núñez, G. K+ Efflux Is the Common Trigger of NLRP3 Inflammasome Activation by Bacterial Toxins and Particulate Matter. Immunity 2013, 38, 1142-1153. [CrossRef]

10. Bauernfeind, F.G.; Horvath, G.; Stutz, A.; Alnemri, E.S.; Macdonald, K.L.; Speert, D.P.; Fernandes-Alnemri, T.; Wu, J.; Monks, B.G.; Fitzgerald, K.A.; et al. Cutting Edge: NF-кB Activating Pattern Recognition and Cytokine Receptors License NLRP3 Inflammasome Activation by Regulating NLRP3 Expression. J. Immunol. 2009, 183, 787-791. [CrossRef]

11. Fernandes-Alnemri, T.; Kang, S.; Anderson, C.; Sagara, J.; Fitzgerald, K.A.; Alnemri, E.S. Cutting Edge: TLR Signaling Licenses IRAK1 for Rapid Activation of the NLRP3 Inflammasome. J. Immunol. 2013, 191, 3995-3999. [CrossRef]

12. Juliana, C.; Fernandes-Alnemri, T.; Kang, S.; Farias, A.; Qin, F.; Alnemri, E.S. Non-transcriptional Priming and Deubiquitination Regulate NLRP3 Inflammasome Activation. J. Biol. Chem. 2012, 287, 36617-36622. [CrossRef]

13. Lin, K.-M.; Hu, W.; Troutman, T.D.; Jennings, M.; Brewer, T.; Li, X.; Nanda, S.; Cohen, P.; Thomas, J.A.; Pasare, C. IRAK-1 bypasses priming and directly links TLRs to rapid NLRP3 inflammasome activation. Proc. Natl. Acad. Sci. USA 2014, 111, 775-780. [CrossRef]

14. Lu, A.; Magupalli, V.G.; Ruan, J.; Yin, Q.; Atianand, M.K.; Vos, M.R.; Schröder, G.F.; Fitzgerald, K.A.; Wu, H.; Egelman, E.H. Unified Polymerization Mechanism for the Assembly of ASC-Dependent Inflammasomes. Cell 2014, 156, 1193-1206. [CrossRef]

15. Masumoto, J.; Taniguchi, S.; Ayukawa, K.; Sarvotham, H.; Kishino, T.; Niikawa, N.; Hidaka, E.; Katsuyama, T.; Higuchi, T.; Sagara, J. ASC, a Novel 22-kDa Protein, Aggregates during Apoptosis of Human Promyelocytic Leukemia HL-60 Cells. J. Biol. Chem. 1999, 274, 33835-33838. [CrossRef]

16. Mariathasan, S.; Weiss, D.S.; Newton, K.; McBride, J.; O’Rourke, K.; Roose-Girma, M.; Lee, W.P.; Weinrauch, Y.; Monack, D.M.; Dixit, V.M. Cryopyrin activates the inflammasome in response to toxins and ATP. Nat. Cell Biol. 2006, 440, 228-232. [CrossRef]

17. Hornung, V.; Bauernfeind, F.G.; Halle, A.; Samstad, E.O.; Kono, H.; Rock, K.L.; Fitzgerald, K.A.; Latz, E. Silica crystals and aluminum salts activate the NALP3 inflammasome through phagosomal destabilization. Nat. Immunol. 2008, 9, 847-856. [CrossRef]

18. Lopez-Castejon, G.; Luheshi, N.M.; Compan, V.; High, S.; Whitehead, R.C.; Flitsch, S.L.; Kirov, A.; Prudovsky, I.; Swanton, E.; Brough, D. Deubiquitinases Regulate the Activity of Caspase-1 and Interleukin-1 $\beta$ Secretion via Assembly of the Inflammasome. J. Biol. Chem. 2013, 288, 2721-2733. [CrossRef]

19. Stutz, A.; Horvath, G.L.; Monks, B.G.; Latz, E. ASC speck formation as a readout for inflammasome activation. Methods Mol. Biol. 2013, 1040, 91-101.

20. Kelley, N.; Jeltema, D.; Duan, Y.; He, Y. The NLRP3 Inflammasome: An Overview of Mechanisms of Activation and Regulation. Int. J. Mol. Sci. 2019, 20, 3328. [CrossRef]

21. Halle, A.; Hornung, V.; Petzold, G.C.; Stewart, C.R.; Monks, B.G.; Reinheckel, T.; Fitzgerald, K.A.; Latz, E.; Moore, K.J.; Golenbock, D.T. The NALP3 inflammasome is involved in the innate immune response to amyloid- $\beta$. Nat. Immunol. 2008, 9, 857-865. [CrossRef]

22. Venegas, C.; Kumar, S.; Franklin, B.S.; Dierkes, T.; Brinkschulte, R.; Tejera, D.; Vieira-Saecker, A.; Schwartz, S.; Santarelli, F.; Kummer, M.P.; et al. Microglia-derived ASC specks cross-seed amyloid- $\beta$ in Alzheimer's disease. Nature 2017, 552, $355-361$. [CrossRef]

23. Hickman, S.E.; Allison, E.K.; El Khoury, J. Microglial Dysfunction and Defective $\beta$-Amyloid Clearance Pathways in Aging Alzheimer's Disease Mice. J. Neurosci. 2008, 28, 8354-8360. [CrossRef]

24. Heneka, M.T. Inflammasome activation and innate immunity in Alzheimer's disease. Brain Pathol. 2017, 27, 220-222. [CrossRef]

25. Ridker, P.M.; Howard, C.P.; Walter, V.; Everett, B.; Libby, P.; Hensen, J.; Thuren, T. Effects of Interleukin-1 $\beta$ Inhibition with Canakinumab on Hemoglobin A1c, Lipids, C-Reactive Protein, Interleukin-6, and Fibrinogen. Circulation 2012, 126, 2739-2748. [CrossRef] 
26. Kitazawa, M.; Oddo, S.; Yamasaki, T.R.; Green, K.N.; LaFerla, F.M. Lipopolysaccharide-Induced Inflammation Exacerbates Tau Pathology by a Cyclin-Dependent Kinase 5-Mediated Pathway in a Transgenic Model of Alzheimer's Disease. J. Neurosci. 2005, 25, 8843-8853. [CrossRef]

27. Sheng, J.G.; Zhu, S.G.; Jones, R.A.; Griffin WS, T.; Mrak, R.E. Interleukin-1 Promotes Expression and Phosphorylation of Neurofilament and tau Proteins in Vivo. Exp. Neurol. 2000, 163, 388-391. [CrossRef]

28. Ising, C.; Venegas, C.; Zhang, S.; Scheiblich, H.; Schmidt, S.V.; Vieira-Saecker, A.; Schwartz, S.; Albasset, S.; McManus, R.M.; Tejera, D.; et al. NLRP3 inflammasome activation drives tau pathology. Nature 2019, 575, 669-673. [CrossRef]

29. Stancu, I.-C.; Cremers, N.; Vanrusselt, H.; Couturier, J.; Vanoosthuyse, A.; Kessels, S.; Lodder, C.; Brône, B.; Huaux, F.; Octave, J.-N.; et al. Aggregated Tau activates NLRP3-ASC inflammasome exacerbating exogenously seeded and non-exogenously seeded Tau pathology in vivo. Acta Neuropathol. 2019, 137, 599-617. [CrossRef]

30. Kitazawa, M.; Cheng, D.; Tsukamoto, M.R.; Koike, M.A.; Wes, P.D.; Vasilevko, V.; Cribbs, D.H.; LaFerla, F.M. Blocking IL-1 Signaling Rescues Cognition, Attenuates Tau Pathology, and Restores Neuronal $\beta$-Catenin Pathway Function in an Alzheimer's Disease Model. J. Immunol. 2011, 187, 6539-6549. [CrossRef]

31. Giuliani, D.; Bitto, A.; Galantucci, M.; Zaffe, D.; Ottani, A.; Irrera, N.; Neri, L.; Cavallini, G.M.; Altavilla, D.; Botticelli, A.R.; et al Melanocortins protect against progression of Alzheimer's disease in triple-transgenic mice by targeting multiple pathophysiological pathways. Neurobiol. Aging 2014, 35, 537-547. [CrossRef]

32. Dempsey, C.; Araiz, A.R.; Bryson, K.; Finucane, O.; Larkin, C.; Mills, E.; Robertson, A.; Cooper, M.; O’Neill, L.; Lynch, M.A. Inhibiting the NLRP3 inflammasome with MCC950 promotes non-phlogistic clearance of amyloid- $\beta$ and cognitive function in APP/PS1 mice. Brain Behav. Immun. 2017, 61, 306-316. [CrossRef]

33. Bitto, A.; Giuliani, D.; Pallio, G.; Irrera, N.; Vandini, E.; Canalini, F.; Zaffe, D.; Ottani, A.; Minutoli, L.; Rinaldi, M.; et al. Effects of COX1-2/5-LOX blockade in Alzheimer transgenic 3xTg-AD mice. Inflamm. Res. 2017, 66, 389-398. [CrossRef]

34. Mirzaei, F.; Khazaei, M.; Komaki, A.; Amiri, I.; Jalili, C. Virgin coconut oil (VCO) by normalizing NLRP3 inflammasome showed potential neuroprotective effects in Amyloid- $\beta$ induced toxicity and high-fat diet fed rat. Food Chem. Toxicol. 2018, 118, 68-83. [CrossRef]

35. Al Rihani, S.B.; Darakjian, L.I.; Kaddoumi, A. Oleocanthal-Rich Extra-Virgin Olive Oil Restores the Blood-Brain Barrier Function through NLRP3 Inflammasome Inhibition Simultaneously with Autophagy Induction in TgSwDI Mice. ACS Chem. Neurosci. 2019, 10, 3543-3554. [CrossRef]

36. Heneka, M.T.; Kummer, M.P.; Stutz, A.; Delekate, A.; Schwartz, S.; Vieira-Saecker, A.; Griep, A.; Axt, D.; Remus, A.; Tzeng, T.C.; et al. NLRP3 is activated in Alzheimer's disease and contributes to pathology in APP/PS1 mice. Nature 2013, 493, 674-678.

37. Goldmann, T.; Tay, T.L.; Prinz, M. Love and death: Microglia, NLRP3 and the Alzheimer's brain. Cell Res. 2013, 23, 595-596. [CrossRef]

38. Lan, Z.; Xie, G.; Wei, M.; Wang, P.; Chen, L. The protective effect of Epimedii Folium and Curculiginis Rhizoma on Alzheimer's disease by the inhibitions of NF-kB/MAPK pathway and NLRP3 inflammasome. Oncotarget 2017, 8, 43709-43720. [CrossRef]

39. Kim, N.; Do, J.; Ju, I.G.; Jeon, S.H.; Kil Lee, J.; Oh, M.S. Picrorhiza kurroa Prevents Memory Deficits by Inhibiting NLRP3 Inflammasome Activation and BACE1 Expression in 5xFAD Mice. Neurotherapeutics 2020, 17, 189-199. [CrossRef]

40. Qiu, W.-Q.; Pan, R.; Tang, Y.; Zhou, X.-G.; Wu, J.-M.; Yu, L.; Law, B.Y.-K.; Ai, W.; Yu, C.-L.; Qin, D.-L.; et al. Lychee seed polyphenol inhibits A $\beta$-induced activation of NLRP3 inflammasome via the LRP1/AMPK mediated autophagy induction. Biomed. Pharmacother. 2020, 130, 110575. [CrossRef]

41. Xiong, R.; Zhou, X.-G.; Tang, Y.; Wu, J.-M.; Sun, Y.-S.; Teng, J.-F.; Pan, R.; Law, B.Y.-K.; Zhao, Y.; Qiu, W.-Q.; et al. Lychee seed polyphenol protects the blood-brain barrier through inhibiting A $\beta(25-35)$-induced NLRP3 inflammasome activation via the AMPK/mTOR/ULK1-mediated autophagy in bEnd.3 cells and APP/PS1 mice. Phytotherapy Res.. [CrossRef]

42. Roy, S. Synuclein and dopamine: The Bonnie and Clyde of Parkinson's disease. Nat. Neurosci. 2017, 20, 1514-1515. [CrossRef]

43. Beraud, D.; Twomey, M.; Bloom, B.; Mittereder, A.; Ton, V.; Neitzke, K.; Chasovskikh, S.; Mhyre, T.R.; Maguire-Zeiss, K.A. $\alpha$-Synuclein Alters Toll-Like Receptor Expression. Front. Behav. Neurosci. 2011, 5, 80. [CrossRef]

44. Gordon, R.; Albornoz, E.A.; Christie, D.C.; Langley, M.R.; Kumar, V.; Mantovani, S.; Robertson, A.A.B.; Butler, M.S.; Rowe, D.B.; $\mathrm{O}^{\prime}$ Neill, L.A.; et al. Inflammasome inhibition prevents $\alpha$-synuclein pathology and dopaminergic neurodegeneration in mice. Sci. Transl. Med. 2018, 10, eaah4066. [CrossRef]

45. Panicker, N.; Saminathan, H.; Jin, H.; Neal, M.; Harishchandra, D.S.; Gordon, R.; Kanthasamy, K.; Lawana, V.; Sarkar, S.; Luo, J.; et al. Fyn Kinase Regulates Microglial Neuroinflammatory Responses in Cell Culture and Animal Models of Parkinson's Disease. J. Neurosci. 2015, 35, 10058-10077. [CrossRef]

46. Panicker, N.; Sarkar, S.; Harishchandra, D.S.; Neal, M.; Kam, T.-I.; Jin, H.; Saminathan, H.; Langley, M.; Charli, A.; Samidurai, M.; et al. Fyn kinase regulates misfolded $\alpha$-synuclein uptake and NLRP3 inflammasome activation in microglia. J. Exp. Med. 2019, 216, 1411-1430. [CrossRef]

47. Mo, Y.; Xu, E.; Wei, R.; Le, B.; Song, L.; Li, D.; Chen, Y.; Ji, X.; Fang, S.; Shen, J.; et al. Bushen-Yizhi Formula Alleviates Neuroinflammation via Inhibiting NLRP3 Inflammasome Activation in a Mouse Model of Parkinson's Disease. Evid.-Based Complement. Altern. Med. 2018, 2018, 1-12. [CrossRef]

48. Han, C.; Guo, L.; Yang, Y.; Li, W.; Sheng, Y.; Wang, J.; Guan, Q.; Zhang, X. Study on antrodia camphorata polysaccharide in alleviating the neuroethology of PD mice by decreasing the expression of NLRP3 inflammasome. Phytotherapy Res. 2019, 33, 2288-2297. [CrossRef] 
49. Han, C.; Shen, H.; Yang, Y.; Sheng, Y.; Wang, J.; Li, W.; Zhou, X.; Guo, L.; Zhai, L.; Guan, Q. Antrodia camphorata polysaccharide resists 6-OHDA-induced dopaminergic neuronal damage by inhibiting ROS-NLRP3 activation. Brain Behav. 2020, $10,01824$. [CrossRef]

50. Feigin, V.; Nichols, E.; Alam, T.; Bannick, M.S.; Beghi, E.; Blake, N.; Culpepper, W.J.; Dorsey, E.R.; Elbaz, A.; Ellenbogen, R.G.; et al. Global, regional, and national burden of neurological disorders, 1990-2016: A systematic analysis for the Global Burden of Disease Study 2016. Lancet Neurol. 2019, 18, 459-480. [CrossRef]

51. Jin, X.; Liu, M.; Zhang, D.; Zhong, X.; Du, K.; Qian, P.; Yao, W.; Gao, H.; Wei, M. Baicalin mitigates cognitive impairment and protects neurons from microglia-mediated neuroinflammation via suppressing NLRP 3 inflammasomes and TLR 4/ NF - $\mathrm{KB}$ signaling pathway. CNS Neurosci. Ther. 2019, 25, 575-590. [CrossRef]

52. Feng, J.; Wang, J.; Du, Y.; Liu, Y.; Zhang, W.; Chen, J.; Liu, Y.; Zheng, M.; Wang, K.; He, G. Dihydromyricetin inhibits microglial activation and neuroinflammation by suppressing NLRP 3 inflammasome activation in APP/PS 1 transgenic mice. CNS Neurosci. Ther. 2018, 24, 1207-1218. [CrossRef]

53. Qi, Y.; Shang, L.; Liao, Z.; Su, H.; Jing, H.; Wu, B.; Bi, K.; Jia, Y. Intracerebroventricular injection of resveratrol ameliorated $\mathrm{A} \beta$-induced learning and cognitive decline in mice. Metab. Brain Dis. 2019, 34, 257-266. [CrossRef]

54. Che, H.; Li, Q.; Liao, W.; Wang, D.; Yang, L.; Xu, J.; Yanagita, T.; Xue, C.; Chang, Y.; Wang, Y.-M. Effects of Astaxanthin and Docosahexaenoic-Acid-Acylated Astaxanthin on Alzheimer's Disease in APP/PS1 Double-Transgenic Mice. J. Agric. Food Chem. 2018, 66, 4948-4957. [CrossRef]

55. Fan, Z.; Liang, Z.; Yang, H.; Pan, Y.; Zheng, Y.; Wang, X.-M. Tenuigenin protects dopaminergic neurons from inflammation via suppressing NLRP3 inflammasome activation in microglia. J. Neuroinflamm. 2017, 14, 1-12. [CrossRef]

56. Dinarello, C.A.; Simon, A.; Van Der Meer, J.W.M. Treating inflammation by blocking interleukin-1 in a broad spectrum of diseases. Nat. Rev. Drug Discov. 2012, 11, 633-652. [CrossRef]

57. Yang, G.; Lee, H.E.; Moon, S.; Ko, K.M.; Koh, J.H.; Seok, J.K.; Min, J.; Heo, T.; Kang, H.C.; Cho, Y.; et al. Direct Binding to NLRP3 Pyrin Domain as a Novel Strategy to Prevent NLRP3-Driven Inflammation and Gouty Arthritis. Arthritis Rheumatol. 2020, 72, 1192-1202. [CrossRef]

58. Yang, G.; Yeon, S.H.; Lee, H.E.; Kang, H.C.; Cho, Y.-Y.; Lee, J.Y. Suppression of NLRP3 inflammasome by oral treatment with sulforaphane alleviates acute gouty inflammation. Rheumatology 2018, 57, 727-736. [CrossRef]

59. Yang, G.; Jang, J.H.; Kim, S.W.; Han, S.-H.; Ma, K.H.; Jang, J.-K.; Kang, H.C.; Cho, Y.-Y.; Lee, H.S.; Lee, J.Y. Sweroside Prevents Non-Alcoholic Steatohepatitis by Suppressing Activation of the NLRP3 Inflammasome. Int. J. Mol. Sci. 2020, 21, 2790. [CrossRef]

60. Song, L.; Pei, L.; Yao, S.; Wu, Y.; Shang, Y. NLRP3 Inflammasome in Neurological Diseases, from Functions to Therapies. Front. Cell. Neurosci. 2017, 11, 63. [CrossRef] 